hep-th/0203213

ITEP-TH-19/02

HUTP-02/A004

\title{
Rational Conformal Field Theories and Complex Multiplication
}

\author{
Sergei Gukov and Cumrun Vafa \\ Jefferson Physical Laboratory \\ Harvard University \\ Cambridge, MA 02138, USA
}

\begin{abstract}
We study the geometric interpretation of two dimensional rational conformal field theories, corresponding to sigma models on Calabi-Yau manifolds. We perform a detailed study of RCFT's corresponding to $T^{2}$ target and identify the Cardy branes with geometric branes. The $T^{2}$ 's leading to RCFT's admit "complex multiplication" which characterizes Cardy branes as specific D0-branes. We propose a condition for the conformal sigma model to be RCFT for arbitrary Calabi-Yau $n$-folds, which agrees with the known cases. Together with recent conjectures by mathematicians it appears that rational conformal theories are not dense in the space of all conformal theories, and sometimes appear to be finite in number for Calabi-Yau $n$-folds for $n>2$. RCFT's on K3 may be dense. We speculate about the meaning of these special points in the moduli spaces of Calabi-Yau $n$-folds in connection with freezing geometric moduli.
\end{abstract}

March 2002 


\section{Introduction}

Two dimensional "Rational Conformal Field Theories" (RCFT) were introduced in [1] as a particularly nice class of conformal field theories which have more structure and could be potentially classified. Moreover it was suggested that perhaps they may be dense in the space of all conformal theories, and so in this way one can potentially get a handle on all conformal theories. RCFT's are characterized by having a symmetry algebra extending the Virasoro algebra (chiral algebra), in terms of which the Hilbert space can be decomposed into finite irreducible representations. Thus RCFT's generalize the notion of minimal models introduced in [2]. Their conjecture motivated a great deal of work on RCFT's leading in particular to Verlinde algebra [3] and the rich structure they encode [4]. Moreover it was shown in [5,6] that RCFT's naturally lead to boundary states, which in modern terminology we call D-brane states.

In the post duality era, we have learned the importance of D-branes in uncovering non-perturbative aspects of string theory. Thus it is natural to ask the following question: We consider strings propagating on a Calabi-Yau background and we vary the moduli of Calabi-Yau. At points on the moduli which correspond to RCFT's we naturally have some finite number of 'special' D-branes. What do these D-branes correspond to? How do we interpret their preferred role at those moduli among the infinitely many allowed D-branes?

Interesting as these question appear, the modern discovery of S-duality raises a further question: Is the notion of RCFT an S-duality invariant concept? The answer to this is no [7]. In fact it could hardly be an S-duality invariant concept because the S-dual theory may not even correspond to string theory so there is no notion of $2 \mathrm{~d}$ conformal theory on the S-dual side. Even if the S-dual theory is a string theory, one can easily see that the RCFT's on one side do not correspond to RCFT's on the dual side. For example type IIB is self-dual, with the roles of fundamental and D-strings exchanged; the rationality of its toroidal compactifications strongly depends on the $B_{N S}$ but is independent of $B_{R}$. However on the S-dual side the roles of $B_{N S}$ and $B_{R}$ are exchanged. Thus rationality is not an S-duality invariant concept. One might thus consider this concept as not being a fundamental concept. However, it turns out that at least in some cases the concept of special points on moduli space makes sense even non-perturbatively. For example the same considerations that apply to rational points on compactification of strings on $T^{2}$ lead to singling out special points on the moduli space of the type IIB string coupling constant $\tau$ which could potentially have some significance in the full non-perturbative theory. Another 
way such a concept may remain relevant non-perturbatively is exemplified by compactification on Calabi-Yau 3-folds. In this case the string coupling constant combines with other hypermultiplets which come from Kähler moduli (in the type IIB case) and so the question of rationality, which seems to split between Kähler and Complex moduli, picks out, in a non-perturbative sense, some special complex moduli on the Calabi-Yau. More generally, at the very least the moduli corresponding to RCFT's must be somehow special at weak string coupling and thus must teach us some extra symmetries about the target space physics.

In this paper we take up the question of RCFT's for sigma models on Calabi-Yau $n$-folds. More specifically we consider the case of complex dimension 1 in detail and use it to advance a conjecture about rational points for the more complicated case of sigma models on Calabi-Yau $n$-folds.

For the case of (complex dimension one) $T^{2}$ target space we uncover the extra symmetry principle for the target space for it to correspond to a RCFT. These correspond to tori which admit Complex Multiplication. This means that there is a complex number $\lambda$ (not real) for which $z \rightarrow \lambda z$ maps $T^{2}$ to itself. This is not necessarily an isomorphism, and is in general a many to one map. Moreover the corresponding Kähler class has to be a complex multiplier $\lambda$ for this to correspond to a RCFT with a diagonal modular invariant. Moreover for each Kähler class there exists a canonical complex multiplication which has the significance that gives the corresponding Cardy states as D0 branes localized at preimages of a given point on $T^{2}$ under this complex multiplication.

In higher dimensions, mathematicians have a generalized notion of complex multiplication [8,9,10], which is natural to conjecture is related to the notion of rationality of conformal theory. This is basically the statement that the mid-dimensional cohomology and the associated variation of Hodge structure, leads naturally to the period matrix of a higher dimensional torus and one asks whether the associated torus admits a complex multiplication. In the case of Calabi-Yau threefolds this leads to a complex torus corresponding to coupling constant matrix of the gauge fields. In the geometric engineering of $\mathcal{N}=2$ theories, this is the associates complex torus encoding the BPS masses of $\mathcal{N}=2$ electric and magnetic charge states. Translated in this way, there is a mathematical conjecture which suggests that in many cases there are only a finite number of points on moduli where the theory is rational. This is in sharp contrast to the case of $T^{2}$ where the rational points are dense in the space of all conformal theories. 
The organization of this paper is as follows: In the next section we review a simple example of $c=1$ RCFT based on a circle, which will help us to introduce the notations and relevant concepts. In section 3 we discuss two families of RCFT based on two-dimensional tori with extra symmetries: $(a)$ direct product of two circles, and $(b)$ a torus with $\mathbb{Z}_{3}$ symmetry. Both examples have been extensively studied in the literature, and we use them to illustrate general features of rational conformal field theories. Section 4 gives a friendly introduction into basics of imaginary quadratic number fields, which play a central role in the characterization of rational CFT's. Following these introductory sections, in section 5 we proceed to the general case of $c=2$ CFT based on elliptic curve, and formulate the criteria for CFT to be rational and, further, to be diagonal. Our results allow to classify such rational conformal field theories. In section 6 we discuss geometric and arithmetic interpretation of Cardy states in RCFT based on elliptic curve. Finally, in section 7 we conjecture a generalization of these results to Calabi-Yau manifolds of higher dimension.

In different contexts, relation between string theory and number theory has been discussed previously in [11, [1, 12, 13, 14, 15, 16].

\section{Review of a Compact Free Boson}

We start with a review of $c=1$ CFT associated with a free bosonic field on a circle of radius $R$. Since this theory was extensively studied in the literature, and has been nicely reviewed in a number of recent papers, see e.g. [17, 18, 19, 20, here we briefly describe only some aspects that will be relevant in the following sections. In our conventions $\alpha^{\prime}=1$, so that the self-dual radius is $R_{s . d}=1$.

For generic values of the radius $R$, the torus partition function has the following form:

$$
Z(q, \bar{q} ; R)=\frac{1}{|\eta|^{2}} \sum_{(p, \bar{p}) \in \Gamma^{1,1}} q^{\frac{1}{2} p^{2}} \bar{q}^{\frac{1}{2} \bar{p}^{2}}
$$

where $q=\exp (2 \pi i \tau)$ and Dedekind's $\eta$-function is defined as

$$
\eta=q^{1 / 24} \prod_{n=1}^{\infty}\left(1-q^{n}\right)
$$

The partition function $Z(q, \bar{q} ; R)$ is given by a sum over the even, self-dual momentum lattice $\Gamma^{1,1}$. Explicitly, the left and the right momenta read:

$$
p=\frac{1}{\sqrt{2}}\left(\frac{n}{R}+m R\right), \quad \bar{p}=\frac{1}{\sqrt{2}}\left(\frac{n}{R}-m R\right)
$$


By definition, the theory is rational if one can represent the partition function $Z(q, \bar{q})$ as a finite sum of the form:

$$
Z(q, \bar{q})=\sum_{j, j} M_{j \bar{j}} \chi_{j}(q) \bar{\chi}_{\bar{j}}(\bar{q})
$$

where $M_{j \bar{j}} \in \mathbb{Z}_{\geq 0}$ and $\chi_{i}$ (resp. $\bar{\chi}_{j}$ ) are holomorphic (resp. anti-holomorphic) characters:

$$
\chi_{j}(q)=\operatorname{tr}_{\mathcal{V}_{j}} q^{L_{0}-c / 24}
$$

For the toroidal examples $\chi_{i}$ and $\bar{\chi}_{j}$ are generalized $\theta$-functions with characteristics.

Modular invariance and existence of the unique vacuum state impose further restrictions on the matrix $M$. Namely, uniqueness of the vacuum implies:

$$
M_{00}=1
$$

On the other hand, modular invariance of the torus partition function requires:

$$
[M, T]=0, \quad[M, S]=0
$$

where matrices $S$ and $T$ determine transformation of the characters under $S L(2, \mathbb{Z})$ :

$$
\begin{aligned}
& \chi_{i}(-1 / \tau)=\sum_{j} S_{i j} \chi_{j}(\tau) \\
& \chi_{i}(\tau+1)=\sum_{j} T_{i j} \chi_{j}(\tau)
\end{aligned}
$$

The matrix $T$ is diagonal and can be written in terms of the conformal dimensions $\Delta_{i}$ and the central charge $c$ :

$$
T_{i j}=\delta_{i j} e^{2 i \pi\left(\Delta_{i}-c / 24\right)}
$$

There is no such simple general expression for the matrix $S$. However, in RCFT's where Verlinde algebra is an abelian group algebra $S_{i j}$ are proportional to roots of unity, which follows from the fact that $S$ diagonalizes Verlinde algebra. For example, in the rational $c=1$ CFT of a compact boson, we have:

$$
S_{j j^{\prime}}=\frac{1}{\sqrt{2 N}} e^{-i \pi j j^{\prime} / N}
$$

For a given RCFT, it is an interesting problem to classify integer matrices $M$, which satisfy the relations (2.4) and (2.5), see [21] for a review. 
Now, let us explain a geometric interpretation of the rationality condition in a theory of a free compact boson. In other words, we want to analyze when the exponent set $\mathcal{I}=\{i\}$ becomes finite. From the explicit form of the left and right momenta (2.2) it is clear that this happens when $R^{2}$ is a rational number:

$$
R^{2}=\frac{k}{l}, \quad k, l \in \mathbb{Z}
$$

where $k$ and $l$ are relatively prime integer numbers. The partition function in this case reads:

$$
Z(q, \bar{q})=\sum_{\substack{i+j=0 \bmod 2 k \\ i-j=0 \bmod 2 l}} \chi_{i}(q) \bar{\chi}_{j}(\bar{q})
$$

It is manifestly invariant under T-duality symmetry, which among other things inverts the radius $R$ and exchanges the winding and momentum modes:

$$
\mathbb{Z}_{2} \quad: \quad R \leftrightarrow \frac{1}{R}, \quad m \leftrightarrow n
$$

The chiral primaries in this theory are labeled by index $j \in \mathbb{Z} \bmod 2 k l$. All theories with the same value of $N=k l$ have the same fusion ring, which in this simple case is just the group algebra of:

$$
\mathbb{Z}_{2 N}
$$

Therefore, theories with the same chiral algebra, but different modular invariants correspond to different ways one can decompose $N$ into a product of two integers. In particular, $R^{2}=$ integer (or $R^{2}=1 /$ integer) correspond to diagonal modular invariants $M_{j \bar{j}}=\delta_{j \bar{j}}$ (or charge conjugation modular invariants):

$$
Z(q, \bar{q})=\sum_{j \in \mathcal{I}} \chi_{j}(q) \bar{\chi}_{j}(\bar{q})
$$

\subsection{Cardy States}

Let us now discuss D-branes in rational conformal field theories. Among all consistent boundary states, there is a special (finite) subset of states, which are invariant under the full extended chiral algebra. This distinguished set of states, called Cardy states, can be systematically constructed in a diagonal RCFT following the original work of Cardy [5].

Let us recall that Cardy states are linear combinations of the Ishibashi states obtained by imposing a modular invariance on the string world-sheet:

$$
\left.|i\rangle=\sum_{i} \frac{S_{i j}}{\sqrt{S_{0 j}}}|j\rangle\right\rangle
$$


In particular, the number of the Cardy states is equal to the number of the Ishibashi states.

The Ishibashi states, in turn, are defined as generalized solutions to the gluing conditions [6]:

$$
\left.\left(W_{n}-(-1)^{\Delta_{W}} \Omega\left(\bar{W}_{-n}\right)\right)|B\rangle\right\rangle=0
$$

where $\Omega$ is a gluing automorphism, and $\Delta_{W}$ is the conformal dimension of the chiral algebra operator $W$. For example, in the case of a free compact boson, the boundary state should preserve the $U(1)$ current $J=i \sqrt{2 N} \partial X$.

Since the Virasoro generators are quadratic in the oscillator modes, the Ishibashi boundary condition (2.11) is solved by:

$$
\left.\left(\alpha_{n}^{\mu}-R_{\nu}^{\mu} \widetilde{\alpha}_{-n}^{\nu}\right)|B\rangle\right\rangle=0
$$

We can also write this condition as:

$$
\partial X^{\mu}(z)=R_{\nu}^{\mu} \bar{\partial} X^{\nu}(\bar{z})
$$

Here we restored space-time indices $\mu, \nu=1, \ldots, d$, and $R$ is an automorphism of the chiral algebra, such that $R \in O(d)$ and $|\operatorname{det}(R)|=1$. In particular, +1 eigenvalues of the automorphism correspond to Neumann boundary conditions, whereas -1 eigenvalues correspond to Dirichlet boundary conditions [17]. For this reason, a boundary state corresponding to an automorphism with $p$ eigenvalues +1 is referred to as a $D p$-brane.

For a given automorphism $R$, the explicit form of the boundary state $|B\rangle\rangle$ satisfying (2.12) is given by:

$$
|B\rangle\rangle=\exp \left[-\sum_{n=1}^{\infty} \frac{1}{n} \alpha_{-n}^{\mu} R_{\mu \nu} \widetilde{\alpha}_{-n}^{\nu}\right] \times \sum|p, \bar{p}\rangle
$$

The sum over momenta in this expression goes over all the elements in the momentum lattice, which satisfy the condition (2.12).

Let us now come back to the case of the diagonal RCFT corresponding to a free boson on a circle of radius $\sqrt{N} R_{s . d .}$. In this case, there are only two choices for the automorphism, $R= \pm 1$, corresponding to D1-branes and D0-branes, respectively. In the latter case, we obtain $2 N$ Ishibashi states, the A-states in the notations of [22,18]:

$$
|A n, n\rangle\rangle=\exp \left[+\sum_{n=1}^{\infty} \frac{1}{n} \alpha_{-n} \widetilde{\alpha}_{-n}\right] \times \sum_{m}\left|\frac{n}{\sqrt{N}}+m \sqrt{N}, \frac{n}{\sqrt{N}}+m \sqrt{N}\right\rangle
$$


where $n \in \mathbb{Z} \bmod 2 N$. For the other automorphism one has only two Ishibashi B-states:

$$
|B n,-n\rangle\rangle=\exp \left[-\sum_{n=1}^{\infty} \frac{1}{n} \alpha_{-n} \widetilde{\alpha}_{-n}\right] \times \sum\left|\frac{n}{\sqrt{N}}+m \sqrt{N},-\frac{n}{\sqrt{N}}-m \sqrt{N}\right\rangle
$$

where $n=0$ or $n=N$.

Using the Cardy's formula (2.10) and the explicit form for the elements of matrix $S$, in this model one finds two boundary states corresponding to D1-branes with different values $( \pm 1)$ of the Wilson line, and $2 N$ D0-branes located at the equidistant positions on the circle, see Figure 1:

$$
\#(\mathrm{Dp}-\text { branes })= \begin{cases}2 N & p=0 \\ 2 & p=1\end{cases}
$$

These Cardy states will be our basic building blocks in a specific example of $c=2$ RCFT discussed next.

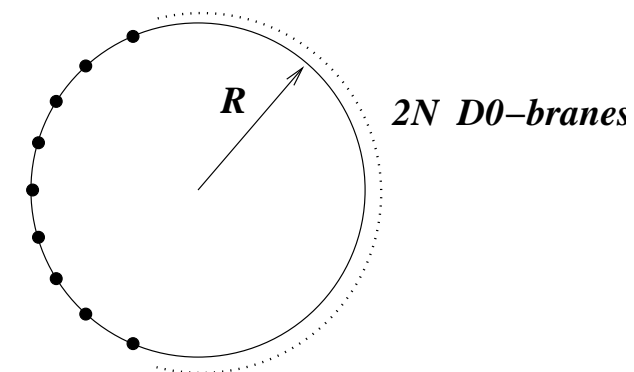

Fig. 1: Cardy states in rational $c=1$ CFT can be identified with equally spaced D0-branes (black dots) on a circle.

\section{Simple Examples of $c=2$ RCFT}

Starting with examples in this section, we proceed to our main subject, namely $c=2$ RCFT based on the elliptic curve $E$. Specifically, we analyze the conditions on the complex structure parameter $\tau$ and the complexified Kähler modulus $\rho$ under which the theory becomes rational and has a diagonal partition function (2.9). These results will help us build some intuition about what should happen in the general case that will be discussed in the following sections. 


\subsection{Basic Notations}

To begin, we summarize our conventions and recall the discrete symmetries of a general RCFT based on the elliptic curve (see also [23] for a nice exposition). Throughout the paper we use the following notations for the real and imaginary parts of $\tau$ and $\rho$ :

$$
\begin{aligned}
& \tau=\tau_{1}+i \tau_{2}=\frac{G_{12}}{G_{11}}+i \frac{\sqrt{\operatorname{det} G}}{G_{11}} \\
& \rho=\rho_{1}+i \rho_{2}=B+i \sqrt{\operatorname{det} G}
\end{aligned}
$$

where $G_{i j}$ denote components of the (flat) metric on $E$. Indeed, it is straightforward to check that

$$
\begin{aligned}
d s^{2} & =\frac{\rho_{2}}{\tau_{2}}|d x+\tau d y|^{2}= \\
& =G_{11} d x^{2}+2 G_{12} d x d y+G_{22} d y^{2}
\end{aligned}
$$

Since we are interested in geometric interpretation of RCFT, we further assume that:

$$
\tau_{2}>0, \quad \rho_{2}>0
$$

RCFT based on the elliptic curve $E$ enjoys a large group of discrete symmetries:

$$
P S L(2, \mathbb{Z})_{\tau} \times P S L(2, \mathbb{Z})_{\rho} \times \mathbb{Z}_{2} \times \mathbb{Z}_{2} \times \mathbb{Z}_{2}
$$

Apart from the last factor, this symmetry group may be viewed as a group of T-dualities. In particular, the first two factors in this group act via modular transformations on $\tau$ and $\rho$, respectively. For example:

$$
P S L(2, \mathbb{Z})_{\tau}: \tau \mapsto \frac{a \tau+b}{c \tau+d}
$$

The $\mathbb{Z}_{2}$ factors, on the other hand, interchange $\tau, \rho$, and their complex conjugates. Specifically, the first $\mathbb{Z}_{2}$ acts as:

$$
\mathbb{Z}_{2}:(\tau, \rho) \mapsto(\rho, \tau)
$$

By analogy with the corresponding symmetry of higher dimensional varieties, we refer to this $\mathbb{Z}_{2}$ as to the mirror transform.

The second $\mathbb{Z}_{2}$ factor in (3.4) is space-time parity transformation acting as follows:

$$
\mathbb{Z}_{2}:(\tau, \rho) \mapsto(-\bar{\tau},-\bar{\rho})
$$


Finally, the last $\mathbb{Z}_{2}$ factor in (3.4) reverses world-sheet orientation:

$$
\mathbb{Z}_{2}:(\tau, \rho) \mapsto(\tau,-\bar{\rho})
$$

\subsection{A Product of Two Circles: $E=\mathbf{S}^{1} \times \mathbf{S}^{1}$}

Our first simple example of $c=2$ RCFT will be a product of two $c=1$ rational CFT's corresponding to a product of two circles of radii:

$$
R_{1}=\sqrt{\frac{k_{1}}{l_{1}}} \text { and } R_{2}=\sqrt{\frac{k_{2}}{l_{2}}}
$$

where $k_{i}$ and $l_{i}$ are some (pairwise co-prime) integers. In these models both $\tau$ and $\rho$ are pure imaginary (modulo real integer part, which can be set to zero by $P S L(2, \mathbb{Z}$ ) transformations):

$$
\tau=i \sqrt{\frac{k_{1} l_{2}}{k_{2} l_{1}}}, \quad \rho=i \sqrt{\frac{k_{1} k_{2}}{l_{1} l_{2}}}
$$

Note, that both $\tau$ and $\rho$ satisfy quadratic equations with integer coefficients (and the same discriminant).

Using the analogous result for a single compact boson, we find that RCFT based $\mathbf{S}^{1} \times \mathbf{S}^{1}$ has a diagonal modular invariant if $l_{1}=1$ and $l_{2}=1$, i.e. up to modular transformations:

$$
\tau=i \sqrt{\frac{k_{1}}{k_{2}}}, \quad \rho=i \sqrt{k_{1} k_{2}}
$$

Another way to describe these $\tau$ and $\rho$ is to say that $\tau$ is a solution to the quadratic equation:

$$
k_{2} \tau^{2}+k_{1}=0
$$

while $\rho$ is an integer multiple of $\tau$ :

$$
\rho=k_{2} \tau
$$

This (strange) relation between $\tau$ and $\rho$ is a precursor of the general property of the elliptic curve, called complex multiplication. As we will see in the following sections, all elliptic curves with this property correspond to a diagonal RCFT, and the converse is also true.

Since in the present example, the RCFT is a product of two theories, the Verlinde algebra is just a product:

$$
\mathbb{Z}_{2 k_{1}} \times \mathbb{Z}_{2 k_{2}}
$$

Note, that the total dimension of the chiral ring is equal to $4 k_{1} k_{2}$. This also gives the number of D0-branes in this theory. Indeed, boundary states corresponding to D-branes in 
the $c=2$ RCFT in consideration can be obtained by tensoring the suitable Cardy states in the two copies of $c=1$ RCFT. Specifically, a product of $\mathrm{D} p_{1}$ boundary state with $\mathrm{D} p_{2}$ boundary state gives a $\mathrm{D}\left(p_{1}+p_{2}\right)$-brane boundary state:

$$
\left|D\left(p_{1}+p_{2}\right)\right\rangle=\left|D p_{1}\right\rangle \otimes\left|D p_{2}\right\rangle
$$

If we tensor two A-type Cardy states, we obtain boundary states corresponding to D0-branes, $4 k_{1} k_{2}$ in number. The D0-branes are distributed on a torus in a regular lattice of $2 k_{1}$ rows and $2 k_{2}$ columns, as shown in Figure 2. On the other hand, if we tensor two B-type boundary states, we get four D2-branes, which cover the entire torus and differ in the values of Wilson lines they carry. Specifically, since each D1-brane on a circle carries \pm 1 Wilson line, by tensoring two of them we get four boundary states, labeled by $( \pm 1, \pm 1)$.

Finally, tensoring A-type Cardy states with B-type Cardy states gives D1-branes parallel to the sides of the torus. Namely, if we tensor D0-brane state on the first circle with a D1-brane state on the second circle, we obtain D1-branes wrapped on the second circle inside $E=\mathbf{S}^{1} \times \mathbf{S}^{1}$. Since we could take $2 k_{1}$ boundary states corresponding to D0-branes and there are 2 possible choices for the Wilson line on the D1-brane, in total we get $2 \times 2 k_{1}=4 k_{1}$ parallel D1-branes. Similar arguments show that there are $4 k_{2}$ parallel D1-branes wrapped on the other basic cycle of the torus, see Figure 2. Hence, there are $4\left(k_{1}+k_{2}\right)$ D1-branes in total.

Summarizing, we have:

$$
\#(\mathrm{Dp}-\text { branes })= \begin{cases}4 k_{1} k_{2} & p=0 \\ 4\left(k_{1}+k_{2}\right) & p=1 \\ 4 & p=2\end{cases}
$$

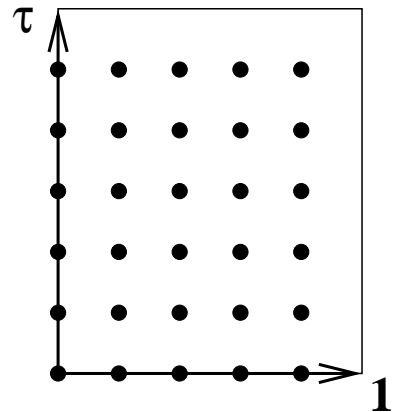

D0-branes

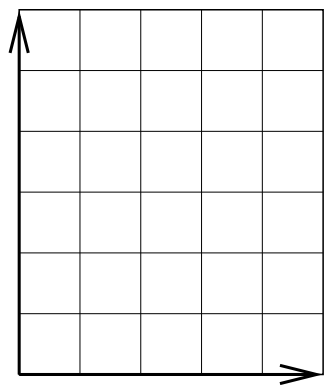

D1-branes

Fig. 2: Cardy states in rational CFT based on a torus $E=\mathbf{S}^{1} \times \mathbf{S}^{1}$. 


\section{3. $S U(3)$ Torus}

There is another simple example of $c=2$ RCFT based on the torus that has been extensively studied in the literature [22,24,25]. It is $S U(3)$ WZW theory at level 1 corresponding to elliptic curve $E$ with extra $\mathbb{Z}_{3}$ symmetry [26]:

$$
\tau=\rho=\exp (2 \pi i / 3)
$$

As in the previous example, in this case both $\tau$ and $\rho$ also satisfy a quadratic equation:

$$
\tau^{2}+\tau+1=0
$$

The dimension of the chiral ring of this theory is equal to 3 , and the Verlinde algebra is just a group algebra of:

\section{$\mathbb{Z}_{3}$}

Comparing this with the previous example, one might expect that in general the fusion rules are given by (a product of) cyclic groups. In what follows we will show that this is indeed always the case; specifically, the Verlinde algebra is a group algebra of:

$$
\mathbb{Z}_{n_{1}} \times \mathbb{Z}_{n_{2}}
$$

This guess includes the special case of a single cyclic group (as in the present example) when one of the factors is trivial, $n_{i}=1$.

Cardy states corresponding to various D-branes in this model have been studied in a number of papers [22,24,25,27]. Here, we summarize the result:

$$
\begin{aligned}
& \#(\mathrm{D} 0-\text { branes })=3 \\
& \#(\mathrm{D} 2-\text { branes })=1
\end{aligned}
$$

The number of D0-branes is expected to be 3 on general grounds. In fact, in all theories the number of Cardy states corresponding to D0-branes should be equal to the dimension of the chiral ring, which is indeed 3 in the present case.

Since generic elliptic curve $E$ does not reduce to a product of two circles, at the moment we have nothing to say about D1-branes. We can just briefly mention that certain boundary states in this model, studied in [22,24, 25, 27], can be identified with D1-branes wrapped on the shortest cycles of the torus rotated by $\pi / 3$, as illustrated on Figure 3 . In the general discussion below we will find the complete set of boundary states in this theory.

A little bit more interesting is a result for D2-branes. Combining it with the result of the previous example, one might conclude that the number of D2-branes can be at least 1 or 4 . Quite surprisingly, we will show that this a general answer: in all $c=2$ RCFT's based on elliptic curve, there is either one or four D2-branes. The number depends on certain arithmetic properties of $E$. 

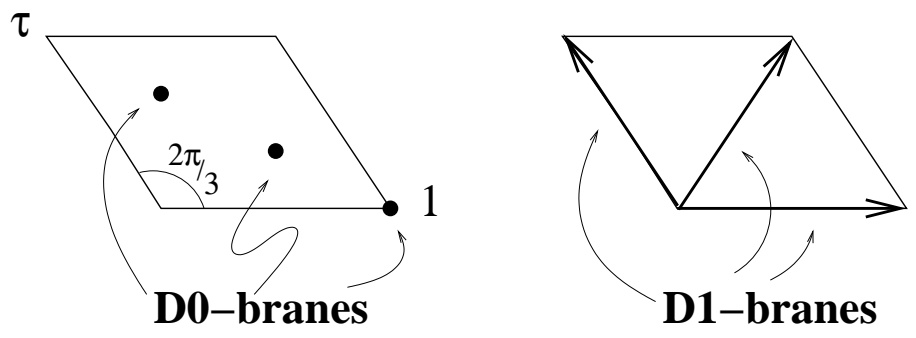

Fig. 3: D-branes on the $S U(3)$ torus.

\section{Imaginary Quadratic Number Fields}

Before we discuss the general case of (rational) conformal field theory based on the elliptic curve $E$ we need to introduce a few basic notions from number theory that will naturally appear in our discussion. In fact, some of these objects have already entered our discussion in the implicit form. For example, in two special cases discussed in the previous section we have noticed that complex parameter $\tau$ satisfies a quadratic equation of the form, $c f$. (3.8) and (3.10):

$$
a \tau^{2}+b \tau+c=0
$$

with relatively prime integer coefficients $a, b$, and $c$. We shall call this quadratic equation a minimal polynomial for $\tau$, and denote by $D$ its discriminant:

$$
D=b^{2}-4 a c
$$

In all cases relevant to physics $D<0$, so that $\tau$ has a non-zero imaginary part:

$$
\tau=\frac{-b+\sqrt{D}}{2 a}
$$

Mathematically, it means that $\tau$ is valued in the imaginary quadratic number field:

$$
K=\mathbb{Q}(\sqrt{D})
$$

This particular way of writing the number field $K$ indicates that it can be obtained from the familiar field of all rational numbers, $\mathbb{Q}$, by introducing $\sqrt{D}$. In other words, every number $x$ in $\mathbb{Q}(\sqrt{D})$ can be written in the form:

$$
x=\alpha+\beta \sqrt{D}
$$


where $\alpha$ and $\beta$ are rational numbers, $\alpha, \beta \in \mathbb{Q}$. Notice, the way we construct the number field $\mathbb{Q}(\sqrt{D})$ from rational numbers is very similar to how one usually defines the field of complex numbers, $\mathbb{C}$, supplementing the field of real numbers, $\mathbb{R}$, with $\sqrt{-1}$.

In our applications, $\tau$ is not just a number - it is a modulus of the elliptic curve:

$$
E=\mathbb{C} /(\mathbb{Z} \oplus \tau \mathbb{Z})
$$

It turns out that elliptic curves with modular parameter $\tau \in \mathbb{Q}(\sqrt{D})$ have a nice property called complex multiplication [28,29, 30]. Elliptic curves with this property enjoy a lot of wonderful arithmetic and geometric properties.

To explain what complex multiplication means, let us consider endomorphisms of the elliptic curve $E$, i.e. holomorphic maps from $E$ to itself:

$$
\varphi \quad: \quad E \rightarrow E
$$

Note, $\varphi$ is a finite degree map (not necessarily degree one). To describe such maps more explicitly, we can view the elliptic curve $E$ as a quotient of a complex plane (parameterized by $z$ ) by a lattice $\mathbb{Z} \oplus \tau \mathbb{Z}, c f$. (4.4). Then, an endomorphism $\varphi$ simply acts as $z \mapsto \varphi z$. Since $\mathbb{Z} \oplus \tau \mathbb{Z}$ is a two-dimensional lattice, we have only have to verify that $\varphi$ maps its generators to some other other elements in this lattice:

$$
\begin{aligned}
& \varphi \cdot 1=m_{1}+n_{1} \tau, \\
& \varphi \cdot \tau=m_{2}+n_{2} \tau
\end{aligned}
$$

Clearly, any elliptic curve has many trivial endomorphisms corresponding to multiplication by an integer, $\varphi \in \mathbb{Z}$. In order to see if there exist any non-trivial endomorphisms, one can take $\varphi$ from the first equation and substitute it to the second equation. As a result, one finds a quadratic equation with integer coefficients of the form (4.1). This simple calculation illustrates that elliptic curves with non-trivial endomorphisms have $\tau$ in some imaginary quadratic field. In fact, it turns out that an elliptic curve $E$ has a non-trivial endomorphism if and only if $\tau$ obeys a quadratic equation (4.1) with integer coefficients. In this case, $E$ is said to have complex multiplication (or to be of CM-type). Summarizing, the endomorphism ring of a general elliptic curve can be one of the following:

$$
\operatorname{End}(E)= \begin{cases}\mathbb{Z}, & \text { no CM } \\ \mathbb{Z}+\mathbb{Z} a \tau, & \text { CM-type, } \tau=\frac{-b+\sqrt{D}}{2 a}\end{cases}
$$


Thus, complex multiplication gives another way to characterize elliptic curves with such 'special' values of $\tau$.

There is a close relation between imaginary quadratic $\tau$, which obey (4.1), and binary quadratic forms:

$$
\left(\begin{array}{cc}
2 a & b \\
b & 2 c
\end{array}\right)
$$

In our discussion, such forms will be associated with intersection form of lattices. Notice, the form above naturally defines a two-dimensional even lattice. However, this form is not unique. Namely, for any $S \in S L(2, \mathbb{Z})$, the intersection form:

$$
\left(\begin{array}{cc}
2 a^{\prime} & b^{\prime} \\
b^{\prime} & 2 c^{\prime}
\end{array}\right)=S\left(\begin{array}{cc}
2 a & b \\
b & 2 c
\end{array}\right) S^{\operatorname{tr}}
$$

defines the same lattice. The invariant associated with such a lattice is the discriminant $D=b^{2}-4 a c$. For a given value of $D$, the equivalence classes of the integral binary quadratic forms form a finite abelian group, the so-called class group [31,28, 32]:

$$
C l(D)
$$

The order of this group $|C l(D)|=h(D)$ is called the class number. It is naturally identified with the number of ideal classes, $h(K)$, of the imaginary quadratic field $K=\mathbb{Q}(\sqrt{D})$.

The last object we need to introduce is an order, $\mathcal{O}_{f}$.

$$
\mathcal{O}_{f}=\mathbb{Z} \oplus \mathbb{Z}[f a \tau]
$$

Here, $a$ a leading coefficient in the quadratic polynomial (4.1) for $\tau$, and $f$ is a positive integer number, called a conductor of $\mathcal{O}_{f}$. We can view an order $\mathcal{O}_{f}$ as a two-dimensional lattice in the number field $K=\mathbb{Q}(\sqrt{D})$, generated by 1 and $f a \tau$. The reason $\mathcal{O}_{f}$ will appear in our discussion is that any element $\varphi \in \mathcal{O}_{f}$ obviously gives a complex multiplication, $c f$. (4.5). Note also, that the endomorphism ring itself, End $(K)$ is also an order with $f=1$.

\section{General $c=2$ RCFT Based on the Elliptic Curve $E$}

In this section we are going to study general $c=2$ conformal field theory based on elliptic curve with arbitrary parameters $\tau$ and $\rho$. We will show that CFT is rational if (and only if) both $\tau$ and $\rho$ take values in the same imaginary quadratic field. The discriminant of this field gives the dimension of the chiral ring. We also show that a condition for diagonal modular invariant implies a further relation between $\tau$ and $\rho$. Namely, one has to be a complex multiplication for the other.

1 This special order in $K$ is called the ring of integers. 


\subsection{Momentum Lattices}

In general, the partition function of $c=2$ CFT based on elliptic curve $E=\mathbb{C} /(\mathbb{Z} \oplus \tau \mathbb{Z})$ :

$$
Z(q, \bar{q})=\frac{1}{\eta^{2} \bar{\eta}^{2}} \sum_{(p, \bar{p}) \in \Gamma^{2,2}} q^{\frac{1}{2} p^{2}} \bar{q}^{\frac{1}{2} \bar{p}^{2}}
$$

is given by the sum over even, self-dual momentum lattice:

$$
\left(\begin{array}{c}
p \\
\bar{p}
\end{array}\right) \in \Gamma^{2,2}=\frac{i}{\sqrt{2 \tau_{2} \rho_{2}}} \mathbb{Z}\left(\begin{array}{l}
1 \\
1
\end{array}\right) \oplus \mathbb{Z}\left(\begin{array}{c}
\bar{\rho} \\
\rho
\end{array}\right) \oplus \mathbb{Z}\left(\begin{array}{c}
\tau \\
\tau
\end{array}\right) \oplus \mathbb{Z}\left(\begin{array}{c}
\bar{\rho} \tau \\
\rho \tau
\end{array}\right)
$$

This lattice will be one of the central objects in our discussion, and, as we shall see in a moment, properties of the CFT, like rationality etc., can be formulated and analyzed in terms of momentum lattices. For this reason, it is convenient to introduce a few more objects associated with the momentum lattice $\Gamma^{2,2}$.

First, we can define the following sublattices in $\Gamma^{2,2}$. Let $\Gamma_{0}$ be the lattice of leftmoving momenta $p$ for a fixed value of $\bar{p}$ (say, for $\bar{p}=0$ ) and a similar lattice $\widetilde{\Gamma}_{0}$ :

$$
\begin{aligned}
& \Gamma_{0}=\left\{p \mid\left(\begin{array}{l}
p \\
0
\end{array}\right) \in \Gamma^{2,2}\right\} \\
& \widetilde{\Gamma}_{0}=\left\{\bar{p} \mid\left(\begin{array}{c}
0 \\
\bar{p}
\end{array}\right) \in \Gamma^{2,2}\right\}
\end{aligned}
$$

Since $\Gamma^{2,2}$ is even integer lattice, the same is true about $\Gamma_{0}$ and $\widetilde{\Gamma}_{0}$. The rank of these lattices is not greater than 2 , and generically it is zero.

By simply forgetting the right (or left) momentum, we can also define the following projections:

$$
\begin{aligned}
& \left.\Gamma_{L}=\left\{p \mid \begin{array}{l}
p \\
*
\end{array}\right) \in \Gamma^{2,2}\right\} \\
& \Gamma_{R}=\left\{\bar{p} \mid\left(\begin{array}{c}
* \\
\bar{p}
\end{array}\right) \in \Gamma^{2,2}\right\}
\end{aligned}
$$

Both $\Gamma_{L}$ and $\Gamma_{R}$ can be characterized as sets where $p$ (or $\bar{p}$ ) take their values. In general, unlike (5.3), $\Gamma_{L, R}$ are not lattices. Of course, in some special cases it may happen that the rank of $\Gamma_{L, R}$ is less than 4 . As we shall see below, these are precisely the occasions relevant to rational theories.

Note, from the above definitions we obtain straightforward relations:

$$
\begin{aligned}
& \Gamma_{0} \subseteq \Gamma_{L} \\
& \widetilde{\Gamma}_{0} \subseteq \Gamma_{R} \\
& \Gamma_{0} \oplus \widetilde{\Gamma}_{0} \subseteq \Gamma^{2,2}
\end{aligned}
$$




\subsection{A Criterion for Rationality}

There are various ways of defining rational CFT's. In our examples associated with tori, it is convenient to formulate the condition for rationality in terms of momentum lattices: a CFT is rational if and only if the left momentum lattice $\Gamma_{0}$ is a finite index sublattice in $\Gamma_{L}$. In such cases, both $\Gamma_{0}$ and $\Gamma_{L}$ are rank two sublattices in $\Gamma^{2,2}$. Moreover, it is easy to see that they are dual lattices:

$$
\Gamma_{0} \cong \Gamma_{L}^{*}, \quad \widetilde{\Gamma}_{0} \cong \Gamma_{R}^{*}
$$

Indeed, since $\Gamma^{2,2}$ is even, self-dual integer lattice, for any vector $(q, \bar{q}) \in \Gamma^{2,2}$ and a given vector $(p, 0) \in \Gamma_{0}$ we have a pairing:

$$
(p, 0) \cdot(q, \bar{q})=p q \in \mathbb{Z}
$$

Therefore, any vector in $\Gamma_{0}$ also belongs to $\Gamma_{L}^{*}$. Conversely, to show $\Gamma_{L}^{*} \subseteq \Gamma_{0}$ let us take a vector $p \in \Gamma_{L}^{*}$. Then, using the above equation and self-duality of $\Gamma^{2,2}$, one finds that $(p, 0)$ is a vector in $\Gamma^{2,2}$. Hence, $\Gamma_{L}^{*} \cong \Gamma_{0}$. Similar arguments give the second isomorphism in (5.6).

Therefore, we conclude that the study of rational conformal field theories based on the elliptic curve $E$ is related to the study of integer even two-dimensional lattices. In particular, it gives a classification of such RCFT's. We will come back to this later.

Now, let us discuss the geometric properties of the elliptic curve corresponding to rational conformal field theory. Suppose that a CFT associated with $E$ is rational, i.e. $\Gamma_{0}$ is a finite index sublattice in $\Gamma_{L}$. Using the explicit expression (5.2) for the right momenta $\bar{p}$, we find that the elements of $\Gamma_{0}$ correspond to integer numbers $\left(m_{1}, m_{2}, n_{1}, n_{2}\right) \in \mathbb{Z}^{4}$, which obey two independent linear relations [7]:

$$
\begin{aligned}
& m_{1}+m_{2} \rho+n_{1} \tau+n_{2} \tau \rho=0 \\
& m_{1}^{\prime}+m_{2}^{\prime} \rho+n_{1}^{\prime} \tau+n_{2}^{\prime} \tau \rho=0
\end{aligned}
$$

If we solve, for example, for $\rho$ from the second equation and substitute the result to the first equation, we find a quadratic equation for $\tau$, with integer coefficients?:

$$
a \tau^{2}+b \tau+c=0, \quad g c d(a, b, c)=1
$$

2 Alternatively, we could use the fact that any three momentum vectors $p \in \Gamma_{L}$ (or $\bar{p} \in \Gamma_{R}$ ) satisfy a linear relation over $\mathbb{Q}$, since $\Gamma_{0}$ is a $\mathbb{Z} \oplus \mathbb{Z}$-module. 
with discriminant $D=b^{2}-4 a c$. Since imaginary part of $\tau$ has to be strictly positive, $c f$. (3.3), we conclude that $\tau$ has to belong to imaginary quadratic number field $K=\mathbb{Q}(\sqrt{D})$ :

$$
\tau=\frac{-b+\sqrt{D}}{2 a}
$$

If we now substitute this $\tau$ back into (5.7), we find that $\rho$ is linear in $\sqrt{D}$ over $\mathbb{Q}$. Hence, both $\tau$ and $\rho$ are elements in $K$. In order to show that the converse is also true, one can take $\tau, \rho \in K$, and construct, for example, $\tau$ and $\tau \rho$ in terms of 1 and $\rho$. Since both $\tau$ and $\rho$ are assumed to be linear functions of $\sqrt{D}$ with rational coefficients (with $\tau_{2}>0$ and $\rho_{2}>0$ ), one can always write $\tau$ and $\tau \rho$ as linear functions of $\rho$ with rational coefficients. Multiplying the resulting relations by a suitable integer, one finds two equations of the form (5.7) with integer coefficients, where $n_{2}=0$ and $n_{1}^{\prime}=0$. Therefore, by construction these relations are independent and define a lattice $\Gamma_{0}$.

Summarizing, we obtain an effective criterion for rationality of $c=2$ conformal field theory based on the elliptic curve $E$ :

$$
\mathrm{RCFT} \Longleftrightarrow \tau, \rho \in \mathbb{Q}(\sqrt{D})
$$

In other words, we found that in order for CFT to be rational, both the target space torus and its dual should have complex multiplication relative to the same quadratic imaginary field.

\subsection{Dimension Of The Chiral Ring And Verlinde Algebra}

As we found in the previous subsection, rational conformal field theories based on the elliptic curve $E$ are naturally attached to even integer lattices. Specifically, let $v_{i}, i=1,2$, be the generators of the momentum lattice $\Gamma_{0}$. Since the intersection form is even, we can write it as:

$$
v_{i} \cdot v_{j}=f\left(\begin{array}{cc}
2 a & b \\
b & 2 c
\end{array}\right)
$$

for some integer numbers $a, b, c$, and $f$, such that $\operatorname{gcd}(a, b, c)=1$.

By definition, the dual lattice $\Gamma_{L}=\Gamma_{0}^{*}$ is generated by vectors $v_{i}^{*}$ with intersection form:

$$
v_{i}^{*} \cdot v_{j}^{*}=\frac{1}{f D}\left(\begin{array}{cc}
-2 a & b \\
b & -2 c
\end{array}\right)
$$

where $D=b^{2}-4 a c$ is the discriminant. It is clear from (5.10) and (5.11) that the dimension of the chiral ring, given by the index $\left[\Gamma_{L}: \Gamma_{0}\right]$, is equal to:

$$
\left[\Gamma_{L}: \Gamma_{0}\right]=f^{2}|D|
$$


Notice, that the right hand side is expressed in terms of invariant quantities.

Furthermore, the Verlinde algebra is the group algebra of:

$$
D\left(\Gamma_{0}\right)=\Gamma_{0}^{*} / \Gamma_{0}
$$

In mathematics literature this group is usually called the discriminant group [33,34, see also [32. It is a finite abelian group of order $f^{2}|D|$. Since $\Gamma_{0}$ is a lattice of rank two, in general, the discriminant group is a product of two cyclic groups, in agreement with what we found in the specific examples of tori with extra symmetries, $c f$. (3.11). Specifically, $D\left(\Gamma_{0}\right)$ is generated by two elements, $g$ and $h$, such that:

$$
g^{2 a f} h^{b f}=1 \quad \text { and } \quad g^{b f} h^{2 c f}=1
$$

The structure of this group depends in a crucial way on the arithmetic. Specifically,

$$
D\left(\Gamma_{0}\right)= \begin{cases}\mathbb{Z}_{f} \times \mathbb{Z}_{f D}, & D=1 \bmod 4 \\ \mathbb{Z}_{2 f} \times \mathbb{Z}_{2 f D^{\prime}}, & D=0 \bmod 4\left(D^{\prime}=D / 4\right), b \neq 0 \\ \mathbb{Z}_{2 f a} \times \mathbb{Z}_{2 f c}, & b=0\end{cases}
$$

This gives a general characterization of the Verlinde algebra in the rational conformal field theory based on the elliptic curve $E$.

\subsection{Diagonal Modular Invariants}

Now we turn to the main problem, namely, analysis of the conditions under which the partition function (5.1) takes the diagonal form (2.9). As we explained in the previous subsections, curve $E$ associated with a rational CFT has complex multiplication. However, the ring $\operatorname{End}(E)$ itself did not enter our discussion so far. Here, we show that RCFT has a diagonal modular invariant iff either $\rho$ or $\tau$ (or modular transformations thereof) belong to $\operatorname{End}(E)$, i.e. iff $\rho$ is a complex multiplication for a given $\tau$, up to discrete symmetries (3.4).

Diagonal modular invariant essentially implies identification of left and right momentum lattices. Namely, given an even integer lattice $\Gamma_{0}$, one can canonically reconstruct the whole momentum lattice $\Gamma^{2,2}$, which is even and self-dual. Specifically, we take two copies of $\Gamma_{L}=\Gamma_{0}^{*}$ :

$$
\Gamma^{2,2}=\left(\Gamma_{L}, \Gamma_{L}^{\prime}\right)
$$

with the equivalence relation:

$$
\Gamma_{L}-\Gamma_{L}^{\prime}=\Gamma_{0}
$$


to see that the lattice (5.13) constructed in this way is even, let us take a vector $(p, \bar{p}) \in \Gamma^{2,2}$. By the equivalence relation (5.14) we have $\bar{p}=p+v$, where $v \in \Gamma_{0}$, and $p \in \Gamma_{0}^{*}$. Therefore,

$$
\begin{aligned}
(p, \bar{p}) \cdot(p, \bar{p}) & =p^{2}-\bar{p}^{2}=(p+\bar{p})(p-\bar{p})= \\
& =-v(2 p+v)=-2 v p-v^{2}
\end{aligned}
$$

Since $\Gamma_{0}$ is taken even, both terms here are even and the claim follows. Furthermore, to show that $\Gamma^{2,2}$ constructed in (5.13) is self-dual, one can provide a basis, $\left(p_{i}, \bar{p}_{i}\right)=$ $\left\{\left(v_{i}^{*}, v_{i}^{*}\right),\left(v_{j}, 0\right)\right\}$, where $v_{j}$ are the generators of $\Gamma_{0}$, and $v_{i}^{*} \in \Gamma_{0}^{*}$ are the dual generators. Then, the bilinear form looks like

$$
\left(p_{i}, \bar{p}_{i}\right) \cdot\left(p_{j}, \bar{p}_{j}\right)=\left(\begin{array}{ll}
0 & \mathbf{1} \\
\mathbf{1} & *
\end{array}\right)
$$

The determinant of this matrix is clearly equal to 1 . Therefore, $\Gamma^{2,2}$ is self-dual.

Now we want to compare a general lattice of the form (5.11) with the momentum lattice (5.2). Up to discrete symmetries (3.4) we can choose $\tau$ and $\rho$, such that the left momentum lattice is generated by (vectors proportional to) 1 and $\tau$ :

$$
\Gamma_{0}^{*}=\Gamma_{L}=\left\{\frac{i}{\sqrt{2 \tau_{2} \rho_{2}}}, \frac{i \tau}{\sqrt{2 \tau_{2} \rho_{2}}}\right\}
$$

It is easy to compute the intersection form of this lattice:

$$
v_{i}^{*} \cdot v_{j}^{*}=\left(\begin{array}{cc}
\frac{1}{2 \tau_{2} \rho_{2}} & \frac{\tau_{1}}{2 \tau_{2} \rho_{2}} \\
\frac{\tau_{1}}{2 \tau_{2} \rho_{2}} & \frac{|\tau|^{2}}{2 \tau_{2} \rho_{2}}
\end{array}\right)
$$

Since $\Gamma_{0}$ has to be an even integer lattice, one should be able to write this intersection as (5.11). Comparing the individual entries, we find that in diagonal RCFT $\tau$ and $\rho$ look like (up to discrete symmetries (3.4)):

$$
\tau=\frac{-b+\sqrt{D}}{2 a}, \quad \rho=f a \tau
$$

Hence, $\rho$ is a complex multiplication for an imaginary quadratic $\tau$. More precisely, $\rho$ should be associated with a generator of an order in the imaginary quadratic field $K=\mathbb{Q}(\sqrt{D})$ :

$$
\rho \in \mathcal{O}_{f}
$$

with the conductor $f$. 
It is easy to verify that the converse is also true. Namely, given $\tau$ and $\rho$ of the form (5.16), the corresponding modular invariant is diagonal. Indeed, substituting (5.16) in the formulas for the momentum vectors $(5.2)$, we find that the momentum lattice $\Gamma^{2,2}$ is of the form (5.13). Specifically, we have:

$$
\begin{aligned}
-i \sqrt{2 a f} \tau_{2} p & =m_{1}+m_{2} \bar{\rho}+n_{1} \tau+n_{2} \bar{\rho} \tau= \\
& =\left(m_{1}+f c n_{2}\right)+\left(n_{1}-a f m_{2}+b f n_{2}\right) \tau
\end{aligned}
$$

It immediately follows that:

$$
\Gamma_{L}=\left\{(p, *)^{T} \in \Gamma^{2,2}\right\}=\frac{i}{\sqrt{2 a f} \tau_{2}}(\mathbb{Z} \oplus \mathbb{Z} \tau)
$$

In order to find elements of $\Gamma_{0}$, we have to solve $\bar{p}=0$. For the right momenta we find:

$$
\begin{aligned}
-i \sqrt{2 a f} \tau_{2} \bar{p} & =m_{1}+m_{2} \rho+n_{1} \tau+n_{2} \rho \tau= \\
& =\left(m_{1}-f c n_{2}\right)+\left(n_{1}+a f m_{2}-b f n_{2}\right) \tau
\end{aligned}
$$

Hence, $\bar{p}=0$ gives two conditions:

$$
\left\{\begin{array}{l}
m_{1}=f c n_{2} \\
n_{1}=-a f m_{2}+b f n_{2}
\end{array}\right.
$$

Substituting this into (5.17) yields:

$$
\left.p\right|_{\bar{p}=0}=\frac{i f}{\sqrt{2 a f} \tau_{2}}\left((2 a \tau+b)\left(-m_{2}\right)+(2 c+b \tau) n_{2}\right) \in \Gamma_{0}
$$

Therefore, we explicitly constructed $\Gamma_{0}$ :

$$
\Gamma_{0}=\left\{(p, 0)^{T} \in \Gamma^{2,2}\right\}=\frac{i f}{\sqrt{2 a f} \tau_{2}}(\mathbb{Z}[2 a \tau+b] \oplus \mathbb{Z}[2 c+b \tau])
$$

The resulting lattices $\Gamma_{L}, \Gamma_{0}$, and $\Gamma^{2,2}$ are related as in (5.13) - (5.14), so that the corresponding CFT is diagonal. It is straightforward to check this directly computing the partition function (5.1) for these momentum lattices. As a result, one finds a diagonal modular invariant:

$$
Z(q, \bar{q})=\sum_{\omega \in \mathcal{I}} \chi_{\omega}(q) \bar{\chi}_{\omega}(\bar{q})
$$

where the exponent $\omega \in \mathcal{I}$ that labels representations of the chiral algebra can be identified with left momenta, $c f$. (5.12):

$$
\omega \in \Gamma_{L} / \Gamma_{0}
$$


and the characters $\chi_{\omega}(q)$ have the form:

$$
\chi_{\omega}(q)=\frac{1}{\eta^{2}} \sum_{v \in \Gamma_{0}} q^{\frac{1}{2}(v+\omega)^{2}}
$$

Finally, we can combine all the results in this section to conclude that diagonal $c=2$ rational conformal field theories are classified by the following data:

i) discriminant $D=0,1 \bmod 4$ (a negative integer, such that $(-D)$ is square-free);

ii) conductor $f$ (a positive integer);

iii) an element of the class group $C l(D)$.

In terms of this data, the CFT has chiral ring of dimension $|D| f^{2}$.

Suppose, for example, we want to know how many diagonal RCFT's have the chiral ring of dimension 163. Using the above results, we can immediately answer this question. Since $h(163)=1$, the answer is surprisingly simple: there is a unique RCFT with this property. On the other hand, if we asked a similar question about RCFT's with chiral ring of dimension 159, we would find $h(159)=10$ such theories. This simple example illustrates sporadic pattern of RCFT's, which nevertheless can be completely explained by the methods of number theory.

Note, it also follows from our analysis that the set of $c=2$ rational conformal field theories on tori is dense.

\subsection{A Digression: The First Main Theorem of Complex Multiplication}

In this subsection (which can be skipped, especially in a single reading) we digress on another remarkable property of elliptic curve with complex multiplication. Throughout the paper we mainly viewed the elliptic curve as a quotient of $\mathbb{C}$ by a two-dimensional lattice. However, we could also view $E$ as an algebraic curve defines, say, by a Weierstrass polynomial:

$$
E: y^{2}=4 x^{3}-g_{2} x-g_{3}
$$

where $g_{2}$ and $g_{3}$ are related to the modular parameter $\tau$ via invariant $j$-function:

$$
\begin{aligned}
j(\tau) & =\frac{1728 g_{2}^{3}}{g_{2}^{3}-27 g_{3}^{2}}=\frac{\theta_{E_{8}}(\tau)^{3}}{\eta(\tau)^{24}}= \\
& =q^{-1}+744+196844 q+21493760 q^{2}+864299970 q^{3}+\ldots
\end{aligned}
$$

Notice, that the coefficients in power series expansion of the $j$-function are integer numbers. 
Even though $j(\tau)$ is a very non-trivial function of $\tau$, there is a nice characterization of its values for $\tau \in \mathbb{Q}(\sqrt{D})$, known as the first main theorem of complex multiplication. Namely, suppose elliptic curve $E$ has a complex multiplication, i.e. $\tau$ obeys a quadratic equation (4.1). Then, $j(\tau)$ also obeys a polynomial equation with integer coefficients of degree $h$ (where $h=h(D)$ is the class number of the field $\mathbb{Q}(\sqrt{D})$ ):

$$
P(z)=z^{h}+a_{1} z^{h-1}+\ldots+a_{h}=0, \quad a_{i} \in \mathbb{Z}
$$

A solution to such equation is called an algebraic integer 3 . Therefore, $j$-invariant of elliptic curve $E$ with complex multiplication is an algebraic integer and $E$ is naturally defined over the number field $K(j(\tau))$.

Motivated by this nice result, one might expect that a proper criterion for CFT to be rational should be formulated as a condition on the algebraic variety to be defined over the algebraic closure $\overline{\mathbb{Q}}$, obtained from the field $\mathbb{Q}$ by adjoining the roots of all irreducible polynomials like (5.25). It is easy to see, however, that this criterion would be wrong. Indeed, it would predict "too many" RCFT's. For example, in the case of Calabi-Yau manifolds it would predict existence of infinitely many points (which are dense) in the moduli space, whereas in the later sections we will argue to the contrary.

More elementary is to see that the above criterion fails already in the case of the elliptic curve $E$. Indeed, in general, the converse of the first main theorem of complex multiplication is not true, so it can not be formulated as "if and only if" condition. On the other hand, from the analysis of the previous sections, we know that CFT is rational if and only if $E$ has complex multiplication. This demonstrates that the right signature of the rational CFT is complex multiplication, rather than a possibility to define the target space variety over $\overline{\mathbb{Q}}$.

\section{Geometric Interpretation of Cardy States}

In the previous sections we have established a relation between RCFT data and arithmetic of the elliptic curve $E$. Motivated by such a relation, one may wonder if it can be extended to string theory, including D-branes and other non-perturbative objects. Due to

3 The first main theorem of complex multiplication further says that if $z=j(\tau)$ is one of these numbers, then $K(j(\tau))$ is the maximal abelian extension of $K=\mathbb{Q}(\sqrt{D})$, with $\operatorname{Gal}(K(j(\tau)) / K)=$ $C l(\operatorname{End}(E))$ acting transitively on the set of numbers $j(\tau)$ 28, 31. 
their geometric nature, D-branes seem to be especially promising. In the weak coupling limit they can be viewed as submanifolds of $E$ of various (co)dimension.

From the CFT point of view, there are some 'special' D0-branes, which preserve the full chiral algebra. The corresponding boundary states were explicitly constructed by Cardy [5]. Therefore, one could ask: "What is arithmetic/geometric interpretation of the Cardy states?" There are several natural candidates for the answer to this question. For example, once we deal with arithmetic of $E$, one might consider rational points of $E$, i.e. solutions of (5.23) with rational values of the coordinates $x, y \in \mathbb{Q}$. However, these can not correspond to Cardy states. Indeed, the number of rational points on $E$ may be infinite, whereas the number of Cardy states is always finite (and equal to the dimension of the chiral algebra):

$$
\#(\text { Cardy states })=|D| f^{2}
$$

where $D$ is the discriminant of the quadratic polynomial (5.8) for $\tau$, and $f$ is the conductor.

In this section we study D-branes on elliptic curve $E$ with diagonal modular invariant:

$$
Z(q, \bar{q})=\sum_{j \in \mathcal{I}} \chi_{j}(q) \bar{\chi}_{j}(\bar{q})
$$

where the exponent $j$ can be identified with momentum, $c f .(5.21)$ :

$$
j \in \Gamma_{L} / \Gamma_{0}
$$

and the characters have the form (5.22). Following Cardy [5], we show that there are always $|D| f^{2}$ D0-branes in this theory, which correspond to the regular points of $\Gamma_{L} / \Gamma_{0}$. On the other hand, the number of D2- and D1-branes depends on the arithmetic of the elliptic curve in a very interesting way. For example,

$$
\#(\mathrm{D} 2-\text { branes })= \begin{cases}1 & \text { Df odd } \\ 4 & \text { Df even }\end{cases}
$$

Note, simple examples studied in section 3 agree with this general result, see (3.9) and (3.12). Below we explain these results in more detail.

Before we start, let us remind that Cardy states are linear combinations of Ishibashi states (2.14):

$$
|B\rangle\rangle=\exp \left[-\sum_{n=1}^{\infty} \frac{1}{n} \alpha_{-n}^{\mu} R_{\mu \nu} \widetilde{\alpha}_{-n}^{\nu}\right] \times \sum|p, \bar{p}\rangle
$$


which satisfy boundary conditions (2.12). It is convenient to write this boundary condition in the following form:

$$
p=-R \bar{p}
$$

Since labels $j$ in $(6.2)$ are identified with momenta, we can say that for a given $R$ the Ishibashi states are labeled by:

$$
(j,-R j), \quad j \in \Gamma_{L} / \Gamma_{0}
$$

However, since we assume that RCFT is diagonal (6.1), the Ishibashi state $(j, \bar{j})$ appears in the closed string spectrum only if $j=\bar{j}$ :

$$
(j, j)=(j,-R j)
$$

It follows that for a given gluing condition (6.4) the Ishibashi states are labeled by fixed points of $R$ in the exponent set $\mathcal{I}$ :

$$
j=-R j, \quad j \in \Gamma_{L} / \Gamma_{0}
$$

In particular, the number of solutions to this conditions give us the number of the corresponding $\mathrm{D} p$-branes. In the following subsections we solve (6.5) for each value of $p$. Note, that $j=0$ is always a solution to $(6.5)$ for any allowed $R$, i.e. there is always at least one corresponding D-brane.

\subsection{D0-branes}

In order to get a D0-brane, we have to impose Dirichlet boundary conditions in both spatial directions, so that $2 \times 2$ matrix $R$ must have two eigenvalues -1 , i.e.:

$$
R=-1
$$

In this case, the boundary condition (6.4) has the

$$
p=\bar{p}
$$

It does not impose any further constraints on the momentum, so that and $p \in \Gamma_{L} / \Gamma_{0}$ is a solution. Hence, the number of D0-branes is given by the following universal formula for all models:

$$
\#(\mathrm{D} 0-\text { branes })=|D| f^{2}
$$


This number is the same as the dimension of the chiral ring, and suggests interpretation of D0-branes as special points on the torus $E$. Indeed, we can think of D0-branes as regular points in the quotient $\Gamma_{L} / \Gamma_{0}$ or else, as preimages of a marked point on a torus $E$ under a specific complex multiplication 1 (see Figure 4):

$$
z \mapsto \widehat{\rho} \cdot z
$$

where, $c f .(5.16)$ :

$$
\widehat{\rho}=f(2 a \tau+b)
$$

Indeed, one can easily check that $\widehat{\rho} \cdot 1=f(2 a \tau+b)$ and $\widehat{\rho} \cdot \tau=-f(2 c+b \tau)$ are the two generators of the lattice $\Gamma_{0}, c f .(5.20)$.

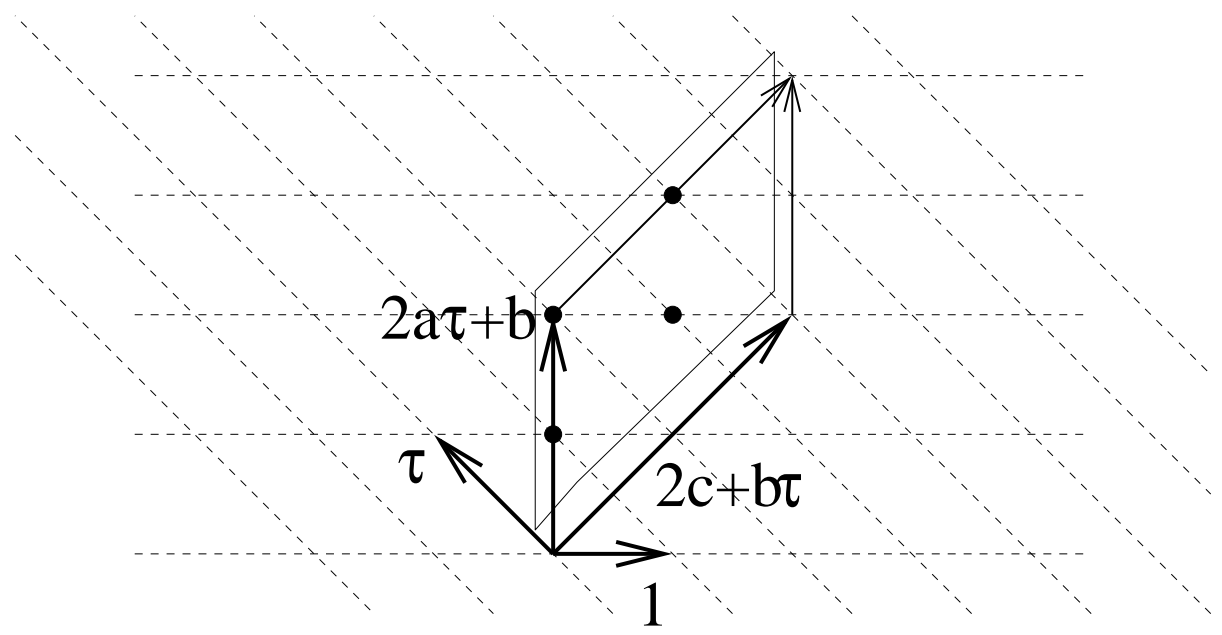

Fig. 4: D0-branes in rational CFT can be identified with points (black dots) in the lattice $\langle 1, \tau\rangle$ modulo $\langle\widehat{\rho}, \widehat{\rho} \tau\rangle$. In this figure we illustrate this for a specific model where $\tau$ satisfies the quadratic equation: $\tau^{2}+2 \tau+2=0$. In this example, $a=1$, $b=2, c=2$, and $D=-4$.

4 The complex multiplication $\widehat{\rho}$ has a number of special properties. First, note that it can be obtained by taking a derivative of the defining quadratic polynomial for $\tau$ :

$$
\widehat{\rho}=f \frac{d Q(\tau)}{d \tau}, \quad Q(\tau)=a \tau^{2}+b \tau+c
$$

Another distinguished property of $\widehat{\rho}$ is that it corresponds to a complex multiplication whose square is multiplication by an integer. Moreover, it is the only complex element (up to integer multiples) in the $\operatorname{End}(E)$ with this property. Explicitly, the square of the element $2 a \tau+b$ is:

$$
(2 a \tau+b)^{2}=4 a^{2} \tau^{2}+4 a b \tau+b^{2}=4 a\left(a \tau^{2}+b \tau\right)+b^{2}=4 a(-c)+b^{2}
$$

which is the discriminant, $D$. 
Geometrically, it is convenient to visualize the set $\Gamma_{L} / \Gamma_{0}$ as a parallelogram with edges $f(2 a \tau+b)$ and $f(2 c+b \tau)$ in the lattice $\Gamma_{L}$.

Note that $\widehat{\rho}=f(2 a \tau+b)$ is closely related to the Kähler structure of the torus, which is $\rho=f a \tau$. In fact, if $b f$ is even $\widehat{\rho}$ can be viewed as twice the Kähler class (with a suitable shift of $\rho$ by $b f / 2$. Thus roughly speaking the Kähler class defines the relevant endomorphism of the torus which defines the Cardy states by its preimage.

\subsection{D2-branes}

The next simplest case is when we impose Neumann boundary conditions in all the directions:

$$
R=1
$$

The involution $R$ inverts the exponent set $\mathcal{I}=\Gamma_{L} / \Gamma_{0}$ :

$$
R \quad: \quad p \mapsto-p
$$

and flips the parallelogram subtended by vectors $f(2 a \tau+b)$ and $f(2 c+b \tau)$, as shown in Figure 5 .
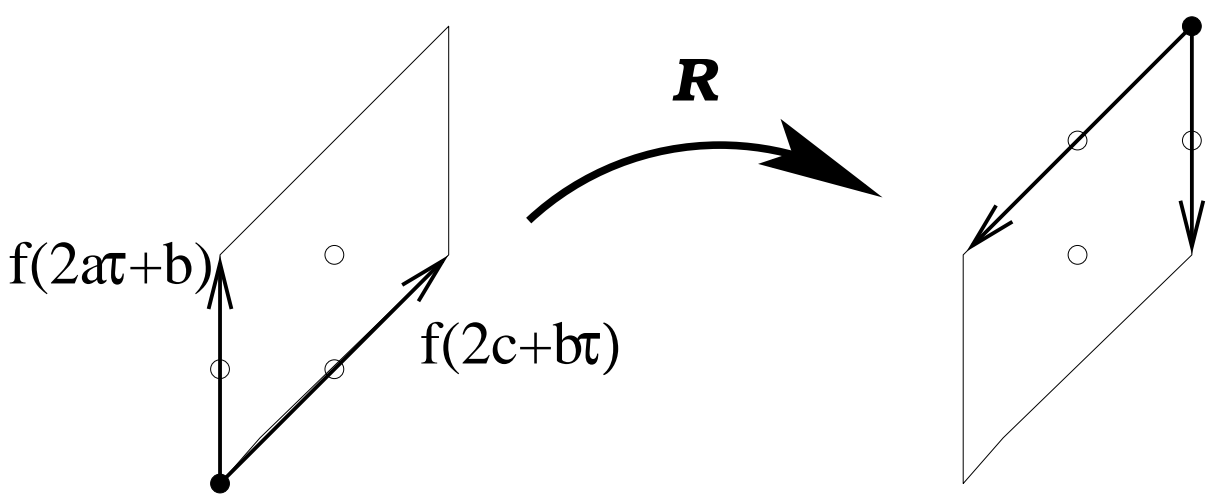

Fig. 5: In the case of D2-brane boundary conditions, the involution $R$ flips the parallelogram made by vectors $f(2 a \tau+b)$ and $f(2 c+b \tau)$. The origin (black dot) is always fixed under this involution. The other three potential fixed points are denoted by empty circles.

According to (6.5), the Ishibashi states are in one-to-one correspondence with the fixed points of this involution, modulo the lattice $\Gamma_{0}$. Geometrically, it is clear that there is either one or four fixed points on the parallelogram (see Figure 5), depending on whether its edges have odd or even coordinates in the lattice $\Gamma_{L} \cong\{1, \tau\}$. Indeed, the origin is 
always a fixed point of $R$. Let us see when there are extra fixed points. The potential candidates are middle points on the edges of the parallelogram and a point in the middle (denoted by empty circles in Figure 5). The explicit coordinates of these points in the lattice $\Gamma_{L} \cong\{1, \tau\}$ are the following:

$$
(f b / 2, f a), \quad(f c, f b / 2), \quad(f c+f b / 2, f a+f b / 2)
$$

It is clear that all of these points are in the lattice $\Gamma_{0}$ if and only if both $f b$ is even. Hence, we arrive to the following general result:

$$
\#(\mathrm{D} 2-\text { branes })= \begin{cases}1 & \text { bf odd } \\ 4 & \text { bf even }\end{cases}
$$

Note that in the case of $b f$ even, the four inequivalent $D 2$ branes differ by the value of $Z_{2}$ Wilson lines along the two cycles.

\subsection{D1-branes}

Finally we consider the case of D1-branes. In this case we have one Neumann and one Dirichlet direction, which correspond to +1 and -1 eigenvalues of $R$, respectively. Allowing for D1-branes of arbitrary orientation, we can write the corresponding involution $R$ as:

$$
R \quad: \quad p \mapsto \alpha p^{*}
$$

where $\alpha$ is some phase, $|\alpha|=1$. Note that this is an order 2 operation. Since $p$ takes values in the lattice $\Gamma_{L} \cong\{1, \tau\}$, the involution $R$ must respect this lattice. In particular it should map a basis of $\Gamma_{L}$ into another basis:

$$
\begin{aligned}
1 & \mapsto-A-B \tau \\
\tau & \mapsto C+D \tau
\end{aligned}
$$

where $A, B, C, D$ are integer numbers, such that $A D-B C=1$. Therefore, we get two conditions:

$$
\begin{aligned}
\alpha & =-A-B \tau \\
\alpha \bar{\tau} & =C+D \tau
\end{aligned}
$$

from which we can eliminate $\alpha$ :

$$
-\bar{\tau}=\frac{A+B \tau}{C+D \tau}
$$

Simply put, the last condition says that $-\bar{\tau}$ should be an involution of $P S L(2 ; \mathbb{Z})$ acting on $\tau$, for otherwise we wouldn't have any D1-branes. Then, for every $\tau$, which satisfy a relation of the form (6.13), there might be different involutions corresponding to different $\alpha$ 's. Therefore, it is natural to split the question in two parts, and classify first all $\tau$ which solve (6.13), and then classify all possible $\alpha$ 's. 


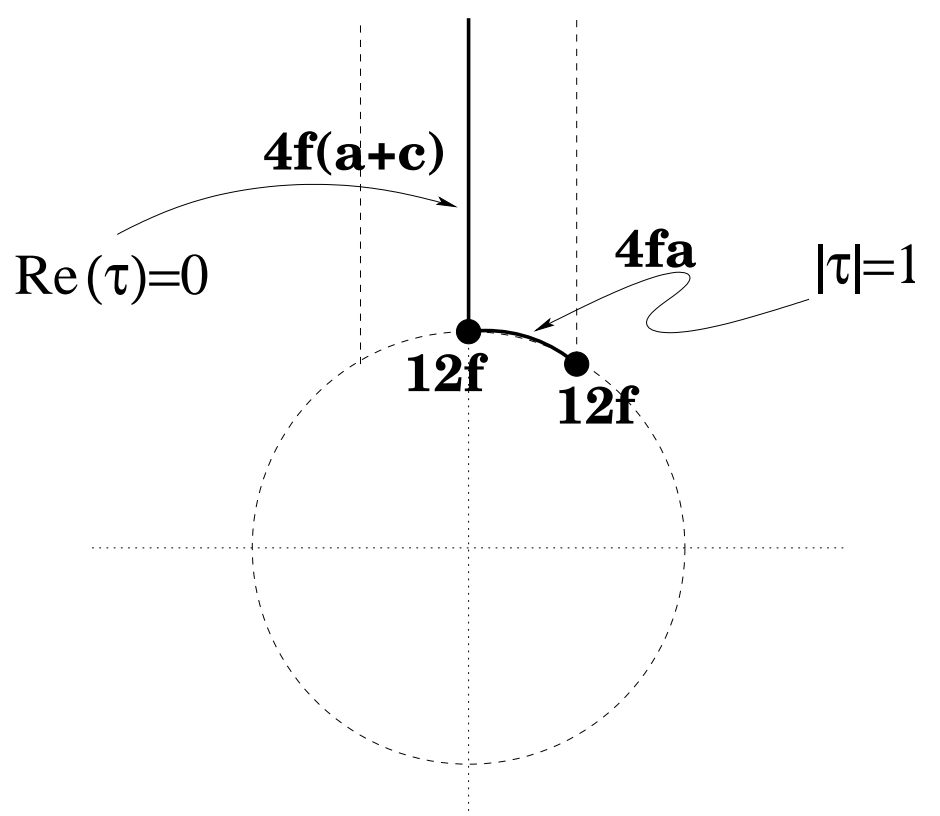

Fig. 6: Two families of the solution for $\tau$ lie either on a unit circle or on the imaginary line. There are also two special cases, $\tau=i$ and $\tau=\exp (2 \pi i / 3)$, represented by black dots. A number near every point indicates the total number of D1-brane boundary states in the corresponding RCFT.

It is easy to see that the only $\tau$ (in the fundamental domain), which solve (6.13), are (i) either pure imaginary, $\tau_{1}=0$, or (ii) lie on a unit circle, $|\tau|=1$, see Figure 6 . To see that these are the only solutions, with no loss of generality we can assume $\tau$ is in the fundamental domain of the upper half plane. We are looking for involutions of $S L(2, Z)$ acting on it which give $-\bar{\tau}$. On the other hand $-\bar{\tau}$ is also in the fundamental domain of the upper half plane. This can only be consistent with the notion of the fundamental domain if the involution is \pm the identity matrix, or it is $\pm S$ in which case it maps the boundary of the fundamental domain to itself. There is one extra case corresponding to the involution $T S T^{-1}$ which fixes $\tau=\exp (2 \pi i / 3)$. In the first case we have $\tau=-\bar{\tau}$ which states that $\tau$ is pure imaginary. In the latter case it implies that $\tau$ is on the unit circle.

The two different families of the solutions for $\tau$ correspond to elliptic curves with different symmetries, simple examples of which were discussed in section 3. Let us now consider each of the two cases in turn:

\section{(i) - a product of two circles}

In the first case $b=0$ and the solutions to (6.13) look like:

$$
\tau=\sqrt{-\frac{c}{a}}
$$


In fact, this is precisely the case discussed in section 3.1, where the torus is a product of two circles:

$$
E=\mathbf{S}^{1} \times \mathbf{S}^{1}
$$

All possible D1-branes in this case were classified in (3.9), with $k_{1}=f a$ and $k_{2}=f c$ :

$$
\#(\mathrm{D} 1-\text { branes })=4 f(a+c)
$$

and come from $\alpha=1$ or $\alpha=-1$. They correspond to $2 f a$ equally spaced $D 1$ branes along one direction and $2 f c$ equally spaced $D 1$ branes along the other direction of the torus. Moreover each of these one branes can have a $\mathbb{Z}_{2}$ Wilson line on them.

\section{(ii) - a torus symmetric relative to the diagonal}

In this case $|\tau|=1$ (which implies $a=c$ ) and the torus $E$ has extra symmetry corresponding to the reflections relative to the diagonals. In particular $z \rightarrow \alpha \bar{z}$ is a symmetry when $\alpha= \pm \tau$, which thus generically yields two involutions $R$. For the case of $\tau=i$ we have 4 involutions given by $z \rightarrow i^{k} \bar{z}$. For $\tau=e^{2 \pi i / 3}$ we have 6 involutions given by $z \rightarrow \omega^{k} \bar{z}$ where $\omega$ is a 6 -th root of unity. The corresponding fixed characters under this involution will correspond to equally spaced D1 branes in the direction given by $\sqrt{\alpha}$.

The corresponding values of $\tau$ are:

$$
\tau=\frac{-b+\sqrt{D}}{2 a}, \quad D=b^{2}-4 a^{2}
$$

For the generic case, the total number of D1-branes, which is just the number of the fixed lattice points under the two reflections is

$$
\#(\mathrm{D} 1-\text { branes })=4 f a
$$

For the case $\tau=i$ the D1 branes making angles multiple of $\pi / 4$. For $D 1$ branes in the direction $2 n \pi / 4(n=0,1)$ we have $2 f$ equally spaced branes each of which can have an extra $Z_{2}$ Wilson line. For $D 1$ branes in the directions $\pi / 4,3 \pi / 4$ we have $2 f$ equally spaced branes (all branes passing through the origin) without any extra possibility of Wilson lines. The $D 1$ branes corresponding to the latter angles do not come from tensoring Ishibashi states of the two decoupled circles, but rather it corresponds to using the extra $Z_{2}$ exchanging the two circles. For $\tau=e^{2 \pi i / 3}$ we have $D 1$ branes which make angles of $2 \pi n / 12$, where $n=0, \ldots, 5$. One can check that for $n$ even there are $3 f$ equally spaced ones and for $n$ odd there are $f$ equally spaced ones. In both special cases $(\tau=i$ and $\tau=\exp (2 \pi i / 3))$ the total number of D1-branes is $12 f$.

We have thus seen that the classification of $D p$ branes for $p>0$ is much more sporadic than those for the $D 0$ branes. This is to be expected because the $D 0$ branes are precisely the ones naturally picked out by the diagonal modular invariant. 


\section{RCFT and Higher Dimensional Calabi-Yau}

So far we have talked about CFT's based on the simplest Calabi-Yau sigma model, namely the target being $T^{2}$. More precisely, we focused only on the bosonic sigma model, but in this case the incorporation of fermions does not modify our discussion, as the fermion partition function is independent of $T^{2}$ moduli. It is natural then to raise the question of RCFT's corresponding to supersymmetric sigma models propagating on higher dimensional Calabi-Yau manifolds.

Unfortunately not much is known about the exact solutions in such cases, and we only have existence proof for such CFT's. The only general classes we know are tensor products of minimal $\mathcal{N}=2$ supersymmetric conformal theories (Gepner models) and toroidal orbifolds. It turns out that both classes are RCFT's or have moduli for which RCFT's appear in a dense subspace. It is the existence of this class of examples which motivates the belief that CFT's are "exactly solvable" if they are rational or near one. Consider quintic threefold, for example. There is only one point in its moduli of Kähler and complex deformation where the CFT is exactly known and that is the RCFT corresponding to the Gepner point. One wonders whether there are other points on the moduli space where they are rational and therefore, perhaps solvable.

In this section we propose a criterion for rationality of conformal theory on Calabi-Yau manifolds which agrees with all the known examples of rational points discussed above (see [35] for a further evidence in the case of toroidal conformal field theories). However, given mathematical conjectures a la André and Oort [36,37] our proposal for rationality suggests that RCFT's are not dense in the generic case of Calabi-Yau sigma models!

Our criterion for rationality is motivated by generalization of the notion of complex multiplication to higher dimensional varieties. Indeed, it was pointed out to us by Kazhdan and Mazur that there already exists a suitable notion of complex multiplication 5 for higher dimensional varieties introduced in 1969 by D. Mumford [8]. In particular, complex multiplication was studied in the context of K3 surface by Piateckii-Shapiro and Shafarevich [9], and more generally, in the context of Calabi-Yau manifolds by Borcea [10. The idea is rather simple: One first defines what it means for an abelian variety (i.e. complex tori) to admit complex multiplication. Then one asks if the variation of the Hodge structure of the Calabi-Yau $M$ and its mirror $W$, whose period matrices lead to a pair of associated abelian varieties admit complex multiplication (of a 'compatible' type).

5 Mathematically, it says that manifold $M$ has complex multiplication when its Hodge group, $\operatorname{Hg}(M)$, is commutative. 


\subsection{Complex Multiplication for Complex Tori}

Consider a complex $n$-dimensional torus.

$$
T^{2 n} \cong \mathbb{C}^{n} / \mathbb{Z}^{2 n}
$$

This is defined by identifications

$$
z_{i} \sim z_{i}+\delta_{i j} \quad z_{i} \sim z_{i}+\mathcal{T}_{i j}
$$

where $\mathcal{T}$ is an $n \times n$ complex symmetric period matrix. Then we say that the torus admits complex multiplication if there exists a non-trivial endomorphism

$$
z \mapsto A z
$$

which implies that

$$
\begin{gathered}
A=M+N \mathcal{T} \\
\mathcal{T} A=M^{\prime}+N^{\prime} \mathcal{T}
\end{gathered}
$$

for some integer matrices $M, N, M^{\prime}, N^{\prime}$. In other words we have a second order matrix equation

$$
\begin{aligned}
& \mathcal{T}(M+N \mathcal{T})=M^{\prime}+N^{\prime} \mathcal{T} \Rightarrow \\
& \mathcal{T} N \mathcal{T}+\mathcal{T} M-N^{\prime} \mathcal{T}-M^{\prime}=0 .
\end{aligned}
$$

Moreover one requires that $N$ has rank $n .6$

\subsection{Calabi-Yau and the Intermediate Jacobian}

The notion of considering mid dimensional cohomology elements and integrating over a mid dimensional integral lattice of cycles to define an abelian variety is well known. For example for the case of a genus $g$ Riemann surface with a symplectic pairing of 1-cycles:

$$
\left(A_{i}, B_{j}\right)=\delta_{i j} \quad\left(A_{i}, A_{j}\right)=\left(B_{i}, B_{j}\right)=0
$$

one considers $g$ holomorphic 1 -forms $\omega_{i}$ normalized relative to the $A$-cycles

$$
\int_{A_{j}} \omega_{i}=\delta_{i j}
$$

6 This rules out examples like $M=E_{C M} \times E^{\prime}$, where $E_{C M}$ is an elliptic curve with complex multiplication and $E^{\prime}$ is another arbitrary elliptic curve without CM. 
and defines the Jacobian by

$$
\int_{B_{j}} \omega_{i}=\mathcal{T}_{i j} .
$$

Similar idea works for arbitrary complex varieties and in particular for Calabi-Yau 3-folds. In the case of Calabi-Yau threefold $\mathcal{T}_{i j}$ can be identified with the complex torus defining the coupling constants of the associated $U(1)^{n}$ gauge fields and is related to the prepotential $\mathcal{F}$ (in the homogeneous coordinates) by

$$
\mathcal{T}_{i j}=\partial_{i} \partial_{j} \mathcal{F}
$$

We say that the Calabi-Yau admits complex multiplication if the corresponding intermediate Jacobian associated with $\mathcal{T}$ admits complex multiplication.

\subsection{A Criterion for RCFT for Calabi-Yau Sigma Models}

A Calabi-Yau sigma model is completely characterized by its complex and Kähler moduli. Since the notion of complex multiplication is natural only for complex structure of the variety, it is natural to associate to a given Calabi-Yau $M$ with a given complex and Kähler structure, a mirror pair of Calabi-Yau $(M, W)$ with fixed complex structures (where we have traded the Kähler structure of the original Calabi-Yau with complex moduli of its mirror). This is effectively how we studied the case of elliptic curve, by viewing $\tau$ and $\rho$ as defining pairs of elliptic curves. We propose the following criterion of the Calabi-Yau sigma model to correspond to a RCFT:

Sigma model on Calabi-Yau corresponding to the pair $(M, W)$ is $R C F T$ if and only if $M$ and $W$ admit complex multiplication over the same number field.

For example, for a Calabi-Yau threefold $M$ satisfying complex multiplication one gets an equation (7.2) of order two in the $\left(h^{2,1}(M)+1\right) \times\left(h^{2,1}(M)+1\right)$ matrix $\mathcal{T}$. In this case, it has been shown by Borcea [10] that existence of complex multiplication is equivalent to the condition that elements of the endomorphism matrix $A$ generate imaginary number field $K$ :

$$
K \cong \operatorname{End}\left(H^{3}(M, \mathbb{Q})\right) \otimes \mathbb{Q}
$$

of degree:

$$
[K: \mathbb{Q}]=2\left(h^{2,1}(M)+1\right)
$$

On the other hand, the mirror $W$ admitting complex multiplication gives elements which are in an algebraic number field of degree $2\left(h^{2,1}(W)+1\right)=2\left(h^{1,1}(M)+1\right)$, and the criterion we are imposing for RCFT is that they are elements of the same number field

\footnotetext{
7 Clearly, the degree of this number field is bounded by $\min \left(2\left(h^{2,1}(M)+1\right), 2\left(h^{1,1}(M)+1\right)\right)$.
} 


\subsection{Application of the Criterion}

To check the criterion, we have to make sure it agrees with the known cases of RCFT's for Calabi-Yau sigma models. Indeed it does. Toroidal orbifolds corresponding to RCFT's obviously admit complex multiplication inherited from the fact that the underlying torus admits complex multiplication (extending our discussion from the elliptic case - the simplest case being orbifolds of the product of elliptic curves). Much more non-trivial are the Gepner points, corresponding to Fermat polynomials. It is also known that these also do admit complex multiplication [38,39,40,10]. Below we show how this works for the quintic threefold with one complex moduli. This is already impressive evidence for the criterion we have proposed for rationality.

We now wish to study how frequently one would encounter rational conformal theory in the moduli of a given Calabi-Yau sigma model, assuming the criterion we have proposed holds. To get a feel for this, consider Riemann surfaces. As discussed above we can identify with it an associated Jacobian. However the moduli space of genus $g$ curves is $3 g-3$ complex dimensional whereas the moduli space of the abelian varieties of dimension $g$ has dimension $g(g+1) / 2$. Thus for $g>4$ the Riemann surfaces are not dense in moduli of the corresponding tori. The Schottky problem is to identify which abelian varieties can arise for Riemann surfaces.

Similarly, one could ask which Riemann surfaces admit complex multiplication. Even though there is a dense set of point in the moduli of complex structure of the tori admitting complex multiplication this may not hold true for the measure zero subspace of it corresponding to those coming from Riemann surfaces. Unlikely as this sounds, indeed there is evidence and a standing mathematical conjecture by Coleman 44] (see also [42] for recent developments) that for sufficiently large $g$ there are only a finite number of Riemann surfaces admitting CM! Indeed a similar conjecture exists for arbitrary varieties 8 and it is believed that the number of CM points are dense only if the relevant moduli space itself is of the form $G / H$ (i.e. a submoduli of the full toroidal moduli defined by some linear

8 Mathematically, a basic version of this conjecture is known as André-Oort conjecture [36, 37], and we thank F. Oort and B. Mazur for explaining to us the general philosophy behind it. Roughly, André-Oort conjecture says that in order for a (sub)family of algebraic varieties to contain a dense set of CM-points, the corresponding moduli space has to be "Shimura (sub)variety". For example, the moduli spaces of elliptic curves and $K 3$ surfaces are of this type, however the moduli space of a Calabi-Yau manifold in general is not. 
algebraic constraint). In particular, in the case of complex tori (of complex dimension $n$ ) and $K 3$ surfaces this conjecture predicts dense set of $\mathrm{CM} / \mathrm{RCFT}$ points. Indeed, in both cases the moduli space turns out to be a coset space:

$$
S O(2 n, 2 n ; \mathbb{Z}) \backslash S O(2 n, 2 n) / S O(2 n) \times S O(2 n)
$$

and

$$
S O(20,4 ; \mathbb{Z}) \backslash S O(20,4) / S O(20) \times S O(4)
$$

respectively. On the other hand, for the case of the one parameter family of quintic threefolds, complex multiplication is conjectured to occur at most at finite number of points. It would be very interesting to test this conjecture as it seems to be at odds with the common lore for RCFT's. This of course might be a blessing in disguise as it seems to point to the existence of some finite number of interesting points on the moduli of CalabiYau compactifications. These may end up being interesting points when the moduli of Calabi-Yau manifolds get frozen by some mechanism.

\subsection{The Example of Fermat Quintic}

Finally, a non-trivial test of our criterion can be obtained by considering a onedimensional family of quintic three-folds

$$
M: \quad z_{1}^{5}+z_{2}^{5}+z_{3}^{5}+z_{4}^{5}+z_{5}^{5}-5 \psi z_{1} z_{2} z_{3} z_{4} z_{5}=0
$$

At the Fermat point, $\psi=0$, the corresponding sigma-model becomes rational, namely it is the $(k=3)^{5}$ Gepner model. On the other hand, Calabi-Yau manifold $M$ has complex multiplication at $\psi=0$ 10,38,39. This is related to the fact that the automorphism group is bigger for the Fermat quintic than for any other generic member in this family. Moreover, $\psi=0$ is the only known non-trivial CM-point in the whole moduli space of $M$. In this sense, there is the same amount of physical and mathematical data on this question, which therefore provides at least one non-trivial check of our proposal.

In order to see explicitly that the Fermat quintic has sufficiently many holomorphic endomorphisms (and, therefore, admits complex multiplication) let us evaluate the period matrix (7.4) at $\psi=0$. In a particular basis of $A$ and $B$ cycles (7.3), the standard calculation gives [13,43]:

$$
\mathcal{T}=\left(\begin{array}{cc}
\alpha-1 & \alpha+\alpha^{3} \\
\alpha+\alpha^{3} & -\alpha^{4}
\end{array}\right)
$$


where $\alpha$ is a (non-trivial) 5 -th root of unity, $\alpha^{5}=1$. Note, that $\alpha$ is a solution to the degree 4 polynomial with integer coefficients:

$$
x^{4}+x^{3}+x^{2}+x+1=0
$$

It is straightforward to check that the matrix $\mathcal{T}$ satisfies the quadratic matrix equation of the form (7.2):

$$
\mathcal{T} N \mathcal{T}+\mathcal{T} M-N^{\prime} \mathcal{T}-M^{\prime}=0
$$

where

$$
N=\left(\begin{array}{cc}
1 & -1 \\
0 & 1
\end{array}\right), \quad M=\left(\begin{array}{ll}
0 & 0 \\
0 & 0
\end{array}\right), \quad N^{\prime}=\left(\begin{array}{cc}
-1 & 0 \\
-1 & 0
\end{array}\right), \quad M^{\prime}=\left(\begin{array}{cc}
-1 & 0 \\
-1 & -1
\end{array}\right)
$$

The corresponding endomorphism is given by the matrix:

$$
A=\left(\begin{array}{cc}
\alpha-1 & \alpha+\alpha^{3} \\
1+\alpha+\alpha^{3} & -\alpha^{4}
\end{array}\right)
$$

Notice, that elements of $\mathcal{T}$ and $A$ take values in a degree 4 number field $K, c f$. (7.5):

$$
K=\mathbb{Q}(\alpha)
$$

which can be obtained from the field of rational numbers, $\mathbb{Q}$, by adjoining the fifth root of unity. Since in the present example $h^{2,1}(M)=1$, this is in complete agreement with the general formula for the degree, $[K: \mathbb{Q}]=2\left(h^{2,1}+1\right)$.

\section{Acknowledgments}

We would like to thank D. Kazhdan and B. Mazur for many illuminating discussions on complex multiplication. We are also grateful to J. de Jong, J. Maldacena, K. Oguiso, H. Ooguri, F. Oort, A. Recknagel, S. Shenker, F. Rodriguez-Villegas, and E. Witten for valuable discussions. This research was partially conducted during the period S.G. served as a Clay Mathematics Institute Long-Term Prize Fellow. The work of S.G. is also supported in part by grant RFBR No. 01-02-17488, and the Russian President's grant No. 00-15-99296. The work of C.V. is supported in part by NSF grants PHY-9802709 and DMS 0074329. 


\section{References}

[1] D. Friedan, Z. Qiu, S. Shenker, "Conformal Invariance, Unitarity, and Two-Dimensional Critical Exponents," Phys. Rev. Lett. 52 (1984) 1575.

[2] A.A. Belavin, A.M. Polyakov, A.B. Zamolodchikov, "Infinite Conformal Symmetry in Two-Dimensional Quantum Field Theory," Nucl. Phys. B241 (1984) 333.

[3] E. Verlinde, "Fusion Rules and Modular Transformations in 2D Conformal Field Theory," Nucl. Phys. B300 (1988) 360.

[4] G. Moore, N. Seiberg, "Polynomial Equations for Rational Conformal Field Theories," Phys. Lett. B212 (1988) 451; "Classical and Quantum Conformal Field Theory," Commun. Math. Phys. 123 (1989) 177; "Taming The Conformal Zoo," Phys. Lett. 220 (1989) 422.

[5] J. L. Cardy, "Boundary Conditions, Fusion Rules And The Verlinde Formula," Nucl. Phys. B 324, 581 (1989).

[6] N. Ishibashi, "The Boundary And Crosscap States In Conformal Field Theories," Mod. Phys. Lett. A4 (1989) 161.

[7] G. Moore, "Arithmetic and attractors," arXiv:hep-th/9807087.

[8] D. Mumford, "A note on Shimura's paper Discontinuous Groups and Abelian Varieties," Math. Ann. 181 (1969) 345.

[9] I. Pjateckii-Shapiro, I.R. Shafarevich, "The Arithmetic of K3 Surfaces," Proc. Steklov Inst. Math. 132 (1973) 45.

[10] C. Borcea, "Calabi-Yau Threefolds and Complex Multiplication," in Essays on Mirror Manifolds, S.-T. Yau ed., International Press, 1992.

[11] B.H. Lian, S.-T. Yau, "Arithmetic Properties of Mirror Map and Quantum Coupling," Commun. Math. Phys. 176 (1996) 163.

[12] S. D. Miller and G. Moore, "Landau-Siegel zeroes and black hole entropy," arXiv:hepth/9903267.

[13] P. Candelas, X. de la Ossa and F. Rodriguez-Villegas, "Calabi-Yau manifolds over finite fields. I," arXiv:hep-th/0012233.

[14] R. Schimmrigk, "Arithmetic of Calabi-Yau varieties and rational conformal field theory," arXiv:hep-th/0111226.

[15] S. Kachru, M. Schulz, S. Trivedi, "Moduli Stabilization from Fluxes in a Simple IIB Orientifold," hep-th/0201028; see also a talk of S. Kachru at Strings 2002 Conference, http://www.damtp.cam.ac.uk/strings02/avt/kachru

[16] Y. Manin, M. Marcolli, "Holography Principle and Arithmetic of Algebraic Curves," hep-th/0201036.

[17] J. Fuchs and C. Schweigert, "Branes: From free fields to general backgrounds," Nucl. Phys. B 530, 99 (1998) arXiv:hep-th/9712257. 
[18] J. Maldacena, G. Moore, N. Seiberg, "Geometrical interpretation of D-branes in gauged WZW models," JHEP 0107 (2001) 046.

[19] A. Cappelli, G. D'Appollonio, "Boundary States of $c=1$ and $c=3 / 2$ Rational Conformal Field Theories," JHEP 0202:039 (2002), hep-th/0201173.

[20] M. R. Gaberdiel and A. Recknagel, "Conformal boundary states for free bosons and fermions," JHEP 0111, 016 (2001) arXiv:hep-th/0108238.

[21] T. Gannon, "Monstrous Moonshine and the Classification of CFT," math.QA/ 9909080 .

[22] H. Ooguri, Y. Oz, Z. Yin, "D-Branes on Calabi-Yau Spaces and Their Mirrors", Nucl.Phys. B477 (1996) 407.

[23] R. Dijkgraaf, E. Verlinde, H. Verlinde, "On Moduli Spaces of Conformal Field Theories with $c \geq 1$," Proc. of 1987 Copenhagen Conference Perspectives in String Theory.

[24] A. Recknagel and V. Schomerus, "D-branes in Gepner models," Nucl. Phys. B 531, 185 (1998) arXiv:hep-th/9712186.

[25] M. Gutperle and Y. Satoh, "D-branes in Gepner models and supersymmetry," Nucl. Phys. B 543, 73 (1999) arXiv:hep-th/9808080.

[26] C. Vafa, "Quantum Symmetries of String Vacua," Mod. Phys. Lett. A4 (1989) 1615.

[27] S. Mizoguchi, T. Tani, "Wound D-Branes in Gepner Models," Nucl. Phys. B611 (2001) 253.

[28] A.N. Parshin, I.R. Shafarevich (Eds.), "Number Theory II," Springer-Verlag, 1992.

[29] G. Shimura, Y. Taniyama, "Complex Multiplication of Abelian Varieties and Its Applications to Number Theory," Japan Math. Soc. 1961.

[30] S. Lang (Ed.), "Number Theory III," Springer-Verlag, 1991.

[31] Z. Borevic, I. Shafarevich, "Number theory," 1985

[32] J.H. Conway, N.J.A. Sloane, "Sphere Packings, Lattices, and Codes," Springer-Verlag, 1993.

[33] V. Nikulin, "Integral symmetric bilinear forms and some of their applications," Math. Izv. 14 (1980) 103.

[34] I. Dolgachev, "Integral quadratic forms: applications to algebraic geometry," Sem. Bourbaki no.611 (1982) 251.

[35] K. Wendland, "Moduli Spaces of Unitary Conformal Field Theories," PhD Thesis, September 2000.

[36] Y. André, "G-functions and Geometry," Aspects of Mathematics, Vol. E13, Vieweg, Braunshweig, 1989; Y. André, "Distribution des points CM sur les sous-variétés de modules de variétés abéliennes, 1997.

[37] F. Oort, "Canonical Lifts and Dense Sets of CM-points," Arithmetic Geometry, Proc. Cortona symposium 1994, F. Catanese, ed., Symposia Math., Vol. XXXVII, Cambridge Univ. Press, 1997, 228. 
[38] T. Shioda, "What is known about the Hodge conjecture?" in Algebraic Varieties and Analytic Varieties, Adv. Studies Pure Math. 1 (1983) 55; T. Shioda, "Geometry of Fermat Varieties," in Number Theory Related to Fermat's Last Theorem, Progress in Math. 26 (1982) 45.

[39] P. Deligne, "Local Behavior of Hodge Structures at Infinity," AMS/IP Studies in Adv. Math. 1 (1997) 683.

[40] P. Deligne (Notes by J. Milne), "Hodge Cycles on Abelian Varieties," Lecture Notes in Math. 900 (1982) 9.

[41] R. Coleman, "Torsion Points on Curves," In Galois representations and arithmetic algebraic geometry (Y. Ihara ed.), Adv. Studies Pure Math. 12 (1987) 235.

[42] J. de Jong, R. Noot, "Jacobians with Complex Multiplication," in Arithmetic Algebraic Geometry (G. van der Geer, F. Oort, J. Steenbrink eds.), Birkhäuser, 1991.

[43] K. Hori, A. Iqbal, C. Vafa, "D-Branes and Mirror Symmetry," hep-th/0005247. 